\title{
TITLE:
}

\section{Laboratory experiments on two coalescing axisymmetric turbulent plumes in a rotating fluid}

\section{AUTHOR(S):}

Yamamoto, H.; Cenedese, C.; Caulfield, C. P.

\section{CITATION:}

Yamamoto, H....[et al]. Laboratory experiments on two coalescing axisymmetric turbulent plumes in a rotating fluid. Physics of Fluids 2011, 23(5): 056601.

ISSUE DATE:

2011

URL:

http://hdl.handle.net/2433/142613

RIGHT:

(C) 2011 American Institute of Physics 


\title{
Laboratory experiments on two coalescing axisymmetric turbulent plumes in a rotating fluid
}

\author{
H. Yamamoto, ${ }^{1, \text { a) }}$ C. Cenedese, ${ }^{2}$ and C. P. Caulfield ${ }^{3,4}$ \\ ${ }^{1}$ Department of Geophysics, Kyoto University, Kyoto, Japan \\ ${ }^{2}$ Department of Physical Oceanography, Woods Hole Oceanographic Institution, Woods Hole, \\ Massachusetts 02543, USA \\ ${ }^{3} B P$ Institute, University of Cambridge, Cambridge, United Kingdom \\ ${ }^{4}$ Department of Applied Mathematics and Theoretical Physics, University of Cambridge, Cambridge, \\ United Kingdom
}

(Received 27 July 2010; accepted 7 April 2011; published online 19 May 2011)

\begin{abstract}
We investigate the early-time coalescence of two co-flowing axisymmetric turbulent plumes and the later-time flow of the induced vortices in a rotating, homogeneous fluid using laboratory experiments. The experiments demonstrate the critical importance of the rotation period $T_{f}=2 \pi / f$, where $f$ is the Coriolis parameter of the background rotation. We find that if the plumes' sources are sufficiently "close" for the plumes to merge initially at an "early time" $t_{m} \lesssim t_{r}=3 T_{f} / 4$, the experimentally observed merging height $z_{m e}$ agrees well with the non-rotating theoretical relationship of $z_{m t} \approx(0.44 / \alpha) x_{0}<z_{r}=5.5 F_{0}^{1 / 4} f^{-3 / 4}$, where $\alpha$ is the entrainment "constant" of the turbulent plumes, $x_{0}$ is the separation distance between the two plume sources, $F_{0}$ is the source buoyancy flux of each plume, and $z_{r}$ is the distance that the plume rises in the time $t_{r}$ before rotational effects become significant. Therefore, rotation does not affect the initial time to merger or the initial merger height of such "close" plumes. For "late" times $t>t_{r}$, however, the flow dynamics are substantially more complicated, as the flow becomes significantly affected by rotation. The propagation and entrainment of the plumes becomes strongly affected by the vortices induced by the entrainment flow in a rotating environment. Also, the plume fluid itself starts to interact with these vortices. If the plumes have already initially merged by the time $t=t_{r}$, a single vortex (initially located at the midpoint of the line connecting the two plume sources) develops, which both advects and modifies the geometry of the merging plumes. Coupled with the various suppressing effects of rotation on the radial plume entrainment, the "apparent" observed height of merger can vary substantially from its initial value. Conversely, for more widely separated "distant" plumes, where $x_{0}>x_{c}=(25 \alpha / 2) F_{0}^{1 / 4} f^{-3 / 4}$, the plumes do not merge before the critical time $t_{r}$ when rotation becomes significant in the flow dynamics and two vortices are observed, each located over a plume source. The combined effect of these vortices with the associated suppression of entrainment by rotation thus significantly further delays the merger of the two plumes, which apparently becomes possible only through the merger of the induced vortices. (C) 2011 American Institute of Physics. [doi:10.1063/1.3584134]
\end{abstract}

\section{INTRODUCTION}

Turbulent buoyant plumes rising from isolated sources occur in a huge range of environmental and industrial contexts. Examples include explosive volcanic eruptions, smoke from chimneys, and seafloor hydrothermal vents. ${ }^{1}$ The "steady" (i.e., either ensemble-averaged or time-averaged over sufficiently long time scales relative to small-scale turbulent fluctuations) dynamics of such plumes are known to be well-described by axisymmetric turbulent plume models, which have attracting similarity solutions in uniform, nonrotating environments. ${ }^{2-5}$ In such circumstances, the "driving" specific buoyancy flux $F_{0}$ remains constant with height, where $F_{0}$ is defined at the source, of radius $R_{s}$ at $z=0$, as

$$
F_{0}=2 \pi \int_{0}^{R_{s}} \frac{g}{\rho_{0}}\left(\rho_{0}-\rho_{s}\right) w_{s} r d r
$$

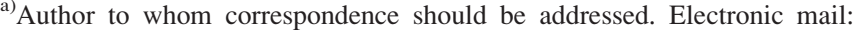
hiroki@kugi.kyoto-u.ac.jp.
}

where $\rho_{0}$ is the ambient fluid density, $\rho_{s}$ and $w_{s}$ are the plume density and vertical velocity at the source, and $r$ is the radial distance from the plume axis. (For simplicity we assume that we may apply the Boussinesq approximation and so $\rho_{0}-\rho_{s} \ll \rho_{0}$, and nevertheless, we also assume that in the distinguished "point source" limit $F_{0}$ remains finite as $R_{s} \rightarrow 0$.)

The behavior of such turbulent plumes is modified by ambient rotation in a subtle fashion, not least because rotation (unlike ambient stratification for example) does not modify $F_{0}$. However, rotation does introduce a key time scale, the inertial period $T_{f}=2 \pi / f$, where $f$ is the Coriolis parameter (twice the system's rotation rate), and so the evolving plume inevitably behaves in a time-dependent manner. At sufficiently early times (much less than the inertial period) in a rotating, homogeneous fluid, the plume initially evolves as if there were no rotation. Fernando et al. ${ }^{6}$ demonstrated that rotation became important after a time $t_{r} \approx 3 T_{f} / 4$. This time $t_{r}$ is usually substantially larger than the time scale of the turbulent fluctuations, and so it is 
legitimate to continue to apply the classical steady model of Morton et $a l .{ }^{2}$ Importantly, Fernando et al. ${ }^{6}$ also demonstrated experimentally that the leading front of a single axisymmetric turbulent plume (or starting plume) propagates as

$$
z \approx 1.7 F_{0}^{\frac{1}{4}} t^{\frac{3}{4}} \text { for } \quad t \leq t_{r} \approx 3 T_{f} / 4
$$

This is the natural relationship on dimensional grounds since $[F]=L^{4} T^{-3}$ and the rotational effects become important only for $t>t_{r} \approx 3 T_{f} / 4$.

As originally discussed by Turner, ${ }^{7,8}$ the starting plume is well-characterized by a leading "thermal" followed by a conical section. Unsurprisingly, since the evolving plume stays well-connected, the rise velocity of both the leading edge thermal and the following conical plume scales in the same way with the source conditions, although due to the fact that the thermal is entraining fluid both from the ambient and the following conical section the thermal is travelling somewhat slower than the rest of the plume on average. Therefore, there is also a characteristic (vertical) distance over which the leading edge of the plume initially rises before the effect of rotation is significant, given by

$$
z_{r} \approx 1.7 F_{0}^{\frac{1}{4}}\left(3 T_{f} / 4\right)^{\frac{3}{4}} \approx 5.5 F_{0}^{\frac{1}{4}} f^{-\frac{3}{4}} .
$$

Up to the time $t_{r}$, or equivalently the distance $z_{r}$ for the leading edge of the plume, we expect the plume dynamics to be largely unaffected by rotation, but then (potentially) markedly modified by rotational effects at later times, as indeed observed by Fernando et al. ${ }^{6}$ Two key interconnected phenomena are that entrainment is suppressed (effectively due to the difficulty of driving an incoming radial entrainment flow into the plume against the prevailing angular momentum distribution) and also that the surviving radially inward entrainment flow is deflected by the Coriolis force, and hence induces a cyclonic circulation (manifested as a largerscale vortex) in the ambient fluid around the rising plume.

To explore the dynamics of an axisymmetric plume, many laboratory experiments with a single plume in various configurations have been carried out in the past (see Table 1 of Ref. 9 for a summary). A particularly interesting problem, with application in both industry and nature, is the coalescence of two relatively close axisymmetric turbulent plumes to form a single plume. Due to entrainment, two nearby plumes will tend to "pull" together, and combine to form a larger "plume" which eventually appears to come from a source with buoyancy flux equal to the sum of the original distinct plume buoyancy fluxes. (Similar dynamics has also been observed in nearby quasi-two-dimensional line plumes, ${ }^{10}$ which are expected to develop below leads in floating ice sheets.) Kaye and Linden ${ }^{11}$ introduced a theoretical model for the merging height of two coalescing axisymmetric turbulent plumes in a non-rotating, homogenous fluid with constant source conditions. Their theory found that the relation between the separation length of the plumes' sources $x_{0}$ and the theoretical merging height $z_{m t}$ is given by

$$
z_{m t} \approx\left(\frac{0.44}{\alpha}\right) x_{0},
$$

where the subscript " $t$ " denotes theoretical prediction for the simplest case of two plumes with the same buoyancy flux, and $\alpha \sim 0.1$ is the conventional entrainment constant relating the effective radial "entrainment velocity" to the characteristic vertical velocity in the plume at the same height. The merging height $z_{m e}$ (" $e$ " for experiment) can be identified in experiments as the height at which the (appropriately averaged) horizontal profile of the density distribution has a single local maximum, and $z_{m e}$ is measured from the "virtual origin." This merger physically manifests itself as the two, initially distinct conical plume bodies combining into one larger plume. Even in the case of two starting plumes, although the above-mentioned leading thermals merge first, the height of merger does not change, and so it is reasonable to think of the merger being associated with the main conical parts of the plumes. This is of course consistent with the experimental technique of using the structure of the density distribution (horizontally averaged in the direction perpendicular to the line connecting the plume sources), as the mechanism to determine the height of merger.

An unambiguous way to define the virtual origin is through consideration of the far-field properties of axisymmetric plumes in a homogeneous unstratified environment. Irrespective of the source conditions, sufficiently far above an actual source, all axisymmetric plumes approach asymptotically a plume in so-called pure plume balance, ${ }^{12}$ where the volume flux $Q$ and (specific) momentum flux $M$ are given in terms of the (constant) specific buoyancy flux $F_{0}$ as

$$
\begin{aligned}
& Q(z) \equiv 2 \pi \int_{0}^{\infty} w_{p}(z, r) r d r=\frac{6 \alpha}{5}\left(\frac{9 \alpha \pi^{2} F_{0}}{10}\right)^{1 / 3}\left(z+z_{v}\right)^{5 / 3}, \\
& M(z) \equiv 2 \pi \int_{0}^{\infty} w_{p}^{2}(z, r) r d r=\left(\frac{9 \sqrt{\pi} \alpha F_{0}}{10}\right)^{2 / 3}\left(z+z_{v}\right)^{4 / 3},
\end{aligned}
$$

where $F_{0}$ is as defined in Eq. (1), $w_{p}$ is the plume vertical velocity (assumed to be self-similar distributions with the same characteristic width for simplicity; for more details see Ref. 5) and $z_{v}$ is the height of the virtual origin. These expressions implicitly define the virtual origin, which may thus be thought of as the notional location of a "point" source of buoyancy flux alone from which the plume appears to be rising at sufficiently large distances above the real source. ${ }^{13}$

Kaye and Linden ${ }^{11}$ also carried out laboratory experiments and showed that their coalescence model is qualitatively correct but over-predicts the merging height slightly. They found that the horizontally averaged density distribution in the plume was very close to Gaussian, consistently with many other observations. ${ }^{1}$ However, as is apparent from Eq. (4), they noted that the quantitative prediction is linearly dependent on the (inverse) value of the entrainment constant, (appropriate for a Gaussian plume) and so a possible explanation of the mismatch is that the entrainment "constant" varies due to the presence of another plume.

The coalescence of turbulent plumes may also occur in nature. For example, chimneys of seafloor hydrothermal vents are typically observed in groups. ${ }^{14}$ Helfrich and Battisti $^{15}$ performed laboratory experiments on the interaction 
of two plume sources in a rotating, stratified fluid. As in a non-rotating flow, the plume overshoots and then collapses back to its neutral buoyancy height (i.e., the height at which the plume density is equal to that of the ambient fluid), where the plume then spreads horizontally, thus generating an anticyclonic circulation. The combination of this anticyclonic circulation with the entrainment-induced cyclonic circulation at lower levels results in an unsteady baroclinic vortex, known as a heton. ${ }^{15}$ They showed that initially hetons are generated, one from each plume, and they repel each other for separations larger than the Rossby radius, i.e.,

$$
x_{0} \geq L_{r}=\frac{N Z_{S}}{f}, \quad N^{2}=-\frac{g}{\rho_{0}} \frac{\partial \rho}{\partial z}
$$

where $N$ is the familiar buoyancy frequency in the Boussinesq approximation, $\rho_{0}$ is a reference density, $Z_{S}$ is the neutral buoyancy level at which the fluid from the plumes initially spreads out, and coalesce for smaller separations. However, the source separation length in their experiments was substantially larger than that considered in Kaye and Linden, ${ }^{11}$ so the coalescence of the plumes themselves did not occur.

In light of this previous work, there is thus a need to consider the effect of rotation on the merger of relatively close plumes. In the present study, we investigate in the laboratory the behavior of two coalescing plumes in a homogeneous fluid with and without background rotation. Our experimental configuration is similar to Kaye and Linden ${ }^{11}$ except for the presence of background rotation in our experiments and differs from Helfrich and Battisti ${ }^{15}$ both in the absence of stratification and in the relatively close source separation, so that it is possible for the plumes themselves to merge. A central issue which must always be considered is the significance of the inherent time-dependence of our experiments. For example, since the influence of rotation appears to become significant after a certain time $t_{r}$, an obvious question to address is whether merger in a rotating environment has the same fundamental character for starting plumes as in the steady non-rotating case, with the merger continuing to occur between the conical parts of the flow, or whether it only occurs at the "thermal" leading edge. We discuss this issue in more detail below.

We describe the experimental procedure in Sec. II, and then present the results of both the non-rotating experiments and the rotating experiments in Sec. III. In particular, we show how the effect of rotation on the merger process manifests itself only at sufficiently late times, and the qualitatively different behavior that occurs for "close" and "distant" plumes, associated with the later-time appearance of one or two vortices. We interpret the vortical motions in light of the dynamics expected in a flow which is significantly influenced by rotation, paying particular attention to the relative importance of the driving buoyancy force of the plume and the Coriolis force. Finally, we present our conclusions in Sec. IV.

\section{EXPERIMENTAL SETUP}

We used a transparent tank with $60 \times 60 \mathrm{~cm}^{2}$ cross section. We filled the tank to a depth of $45 \mathrm{~cm}$ with fresh water

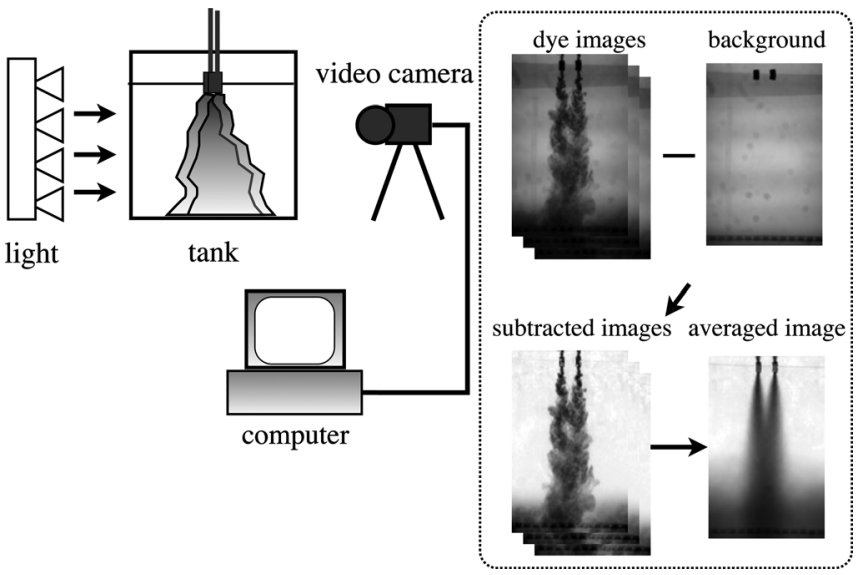

FIG. 1. Schematic illustration of the dye attenuation technique.

$\left(\rho_{0}=1.0 \mathrm{~g} / \mathrm{cm}^{3}\right)$ for the ambient fluid, placed the tank on a rotating table and span the fluid up close to solid body rotation. We then used dyed seawater $\left(\rho_{s}=1.025 \mathrm{~g} / \mathrm{cm}^{3}\right)$ to generate the axisymmetric turbulent plumes.

We placed the plume sources just below the free surface of the ambient water. The plume sources had an opening with diameter $5 \mathrm{~mm}$, and followed the design due to Professor Paul Cooper. ${ }^{11}$ The particular attraction of this design is that, due to an internal small hole opening into a wider chamber, the plume fluid is turbulent on exit from the opening. We kept the source volume flow rate $Q_{s}$ constant for all the experiments, equal to $1.7 \mathrm{~cm}^{3} / \mathrm{s}$. Therefore, the (specific) buoyancy flux, $F_{0}=g Q_{s}$ $\left(\rho_{s}-\rho_{0}\right) / \rho_{0}$, was $41 \mathrm{~cm}^{4} / \mathrm{s}^{3}$. We also mounted video cameras above and on the side of the tank on the rotating table so that we were able to observe the flow in the rotating frame.

We visualized the plumes' behavior (see Fig. 1) using a dye-attenuation technique, and captured a sequence of dye images using side-view video recordings. Using the image processing software Digiflow (http://www.damtp.cam.ac.uk/ lab/digiflow/index.html), we subtracted the background image from the dye images. The grey-scale of the subtracted images indicates the dye concentration which can be correlated directly and accurately to the density (and hence buoyancy) distribution, averaged in the direction of the line of sight, since the light intensity is proportional to the dye concentration. We time-averaged the sequence of subtracted images to obtain an image with a spatially smooth mean distribution of buoyancy. The averaging period was $120 \mathrm{~s}$ for the non-rotating cases and $T_{f} / 4$ for the rotating cases. The capturing time intervals are shown in Table I (see Kaye and Linden ${ }^{11}$ for details of this technique).

TABLE I. Time intervals for capturing dye images.

\begin{tabular}{lc}
\hline \hline$f=2 \Omega\left(\mathrm{s}^{-1}\right)$ & Time interval (s) \\
\hline $0\left(x_{0} \leq 6 \mathrm{~cm}\right)$ & 1 \\
$0\left(x_{0}>6 \mathrm{~cm}\right)$ & 0.25 \\
0.05 & 1 \\
0.1 & 0.5 \\
0.25 & 0.25 \\
0.5 & 0.1 \\
0.75 & 0.1 \\
1 & 0.1 \\
\hline \hline
\end{tabular}




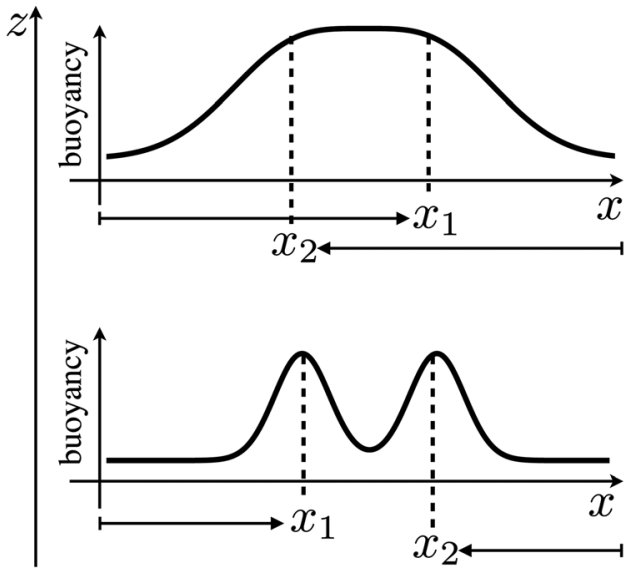

FIG. 2. Location of $x_{1}$ and $x_{2}$ in the non-merging case (lower curve) and the merging case (upper curve). The solid line indicates the horizontal buoyancy profile.

Following Kaye and Linden's approach, ${ }^{11}$ we define the experimentally observed height of merger $z_{m e}$ as the height, where the time-averaged buoyancy profile, horizontally averaged in the direction perpendicular to the line connecting the two plume sources, has a single maximum, measured from the virtual origin. In theory of course, the buoyancy distribution of the appropriately time-averaged image should have only one or two local maximum at every height. However, even in the image averaged over a period of $120 \mathrm{~s}$, there were some fluctuations in the buoyancy profile, due apparently to a combination of turbulence and noise in the signal. Therefore, we developed a thresholding algorithm to determine the merging height unambiguously. The first step of the algorithm involved smoothing the signal by box-averaging. We average over seven pixels for $x_{0} \leq 6 \mathrm{~cm}$ and thirteen pixels for $x_{0}>6 \mathrm{~cm}$, where one pixel corresponds to 0.07 $\mathrm{cm}$. Then, starting from the left, we identify the first location $x_{1}$ where the profile has a significant negative slope. Starting in turn from the right, we also identify the first location $x_{2}$ where the profile has a significant positive slope. If $x_{1}<x_{2}$, the plumes have not merged, as shown schematically in the lower curve of Fig. 2. On the other hand, if $x_{1}>x_{2}$, we consider the plumes to have merged, as shown schematically in the upper curve of Fig. 2. Therefore, comparing $x_{1}$ with $x_{2}$ at every height, the experimentally observed merging height $z_{m e}$ can be defined unambiguously. A more detailed description of the algorithm can be found in Ref. 16. In order to visualize the evolution of the ambient flow induced by the plumes in the rotating experiments, we also colored the free surface of the water with both black and fluorescent yellow powder dyes and recorded the top-view using video, and colored the tank water with potassium permanganate crystals and took still images.

We conducted 23 different non-rotating experiments, varying the separation length $x_{0}$ between 2.4 and $10.3 \mathrm{~cm}$. For our rotating experiments, both the Coriolis parameter $f=2 \Omega(\Omega$ is the angular velocity of the background rotation) and the separation of the plumes' sources $x_{0}$ are important parameters, and so we carried out rotating experiments with combinations of $f=0.05,0.1,0.25,0.5,0.75,1.0 \mathrm{~s}^{-1}$ and $x_{0} \approx 3,5,8,10 \mathrm{~cm}$. In an attempt to make the effect of

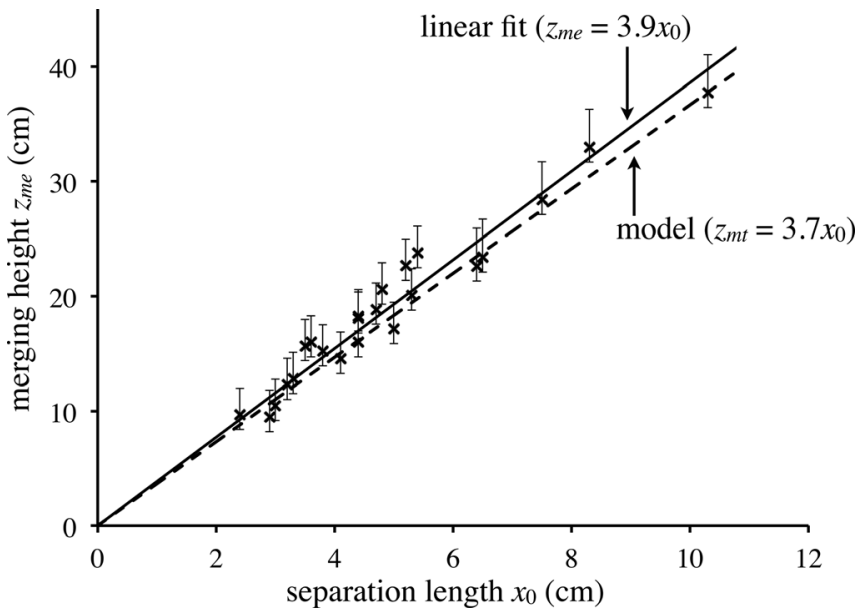

FIG. 3. Experimentally measured plume merging height $z_{m e}$ plotted against the initial separation length $x_{0}$. The solid line is the best linear fit of the experimental data and the dashed line is the theoretical prediction given in Eq. (4), i.e., $z_{m t}=(0.44 / \alpha) x_{0}$ with $\alpha=0.12$. The error bars correspond to errors of $+2.3 /-1.3 \mathrm{~cm}$ for $x_{0} \leq 6 \mathrm{~cm}$ and $+3.3 /-1.3 \mathrm{~cm}$ for $x_{0}>6 \mathrm{~cm}$, errors which are estimated to arise from the sum of the standard deviation of $z_{m e}$ and the one-sided error inherent in box-averaging of the images.

rotation as similar as possible for each plume, we endeavoured to place the plume sources equidistant from and collinear with the intersection of the rotation axis with the free surface in the experimental tank.

\section{EXPERIMENTAL RESULTS}

\section{A. Non-rotating experiments $(f=0)$ : Merging height}

For the non-rotating experiments, Fig. 3 shows the value of $z_{m e}$ plotted against $x_{0}$. The solid line is the linear fit of our experimental data and the dashed line is the theoretical prediction for $z_{m t}$ (Eq. (4)), where we have included the effect of the virtual origin, which in the present study, we determined to be $z_{v}=1.0 \pm 0.5 \mathrm{~cm}$. We determined $z_{v}$ and the entrainment constant $\alpha=0.12 \pm 0.01$ associated with our plume sources by using the direct "filling box" technique ${ }^{17}$ for a single plume. We estimated the displayed error bars as the sum of the standard deviation of $z_{m e}$ and the (systematic) error associated with box-averaging. We calculated the standard deviation of $z_{m e}(\simeq 1.3 \mathrm{~cm})$ by repeating experiments with the same separation length, and identifying $z_{m e}$ in an image averaged over a period of $120 \mathrm{~s}$. The systematic error inherent in box-averaging is inevitable and one-sided, since box-averaging may combine two peaks which are close yet still distinct into one spurious peak, but never separate a single peak into two spurious peaks. From consideration of the number of boxes over which we box-averaged, we estimated this (one-sided) error as being approximately $1 \mathrm{~cm}$ for $x_{0} \leq 6 \mathrm{~cm}$ and $2 \mathrm{~cm}$ for $x_{0}>6 \mathrm{~cm}$.

Our experimental results do indeed show a linear relationship between $z_{m e}$ and $x_{0}$, in agreement with the theoretical prediction, with a difference between the best linear fit $\left(z_{m e}\right.$ $\left.=3.9 x_{0}\right)$ and the model (Eq. (4)) with $\alpha=0.12\left(z_{m t}=3.7 x_{0}\right)$ corresponding to a $10 \%$ error in the entrainment constant. This error is certainly within the typical range of experimental uncertainty, especially since the independent measurement 
of $\alpha$ was done for a non-interacting plume. Consistently with the observations of Kaye and Linden, ${ }^{11}$ merger continued to occur at the conical plume-like part of a starting plume once the initial fronts had departed, and so did not depend on the properties of the leading-edge thermals. ${ }^{7}$

\section{B. Front evolution}

To understand the evolution of the plume-merging flow in a rotating environment, it is very useful to verify the front evolution relation (Eq. (2)), remembering that, although this is for leading "thermal," we expect the following conical plume-like part of the flow to have the same characteristic scaling. Therefore, we tracked the location of the leading front of a plume for relatively short times (i.e., for times $t<t_{r}=3 T_{f} / 4$ ). We found that in both rotating and non-rotating experiments, the location of the leading front approximately agrees with (Eq. (2)), as shown in Fig. 4. In particular, this allows us to use (Eq. (3)) to predict the depth at which rotation is expected to affect the plumes' dynamics significantly. It is important to appreciate that we effectively only verified the insensitivity to the Coriolis parameter $f$ at these early times, since we did not vary the source buoyancy flux $F_{0}$ of the plume.

\section{Rotating experiments $(f>0)$ : Merging height}

Armed with this knowledge that the evolution of the leading front of the plume is largely unaffected by rotation below the height $z_{r}$, or equivalently until the time $t_{r}$, we investigated the initial merging height $z_{m e}$ of plumes which were relatively close together, i.e., where $z_{m e}<z_{r}$. The initial merging time identified in a non-rotating environment is expected to occur before rotational effects become significant, and so $t_{m}<t_{r}$. For the rotating experiments, in Fig. 5, we show the relation between $z_{m e}$ and $x_{0}$ for $T_{f} / 2<t_{m}$ $<t_{r}=3 T_{f} / 4$ with solid symbols, where $t_{m}$ is the time to merge initially. We estimated the error bars in a similar way to that used for Fig. 3. However, in this case, the requirement to focus on relatively early time dynamics largely unaffected by rotation meant that we used a shorter time interval over which to average the images than in the estimation of the

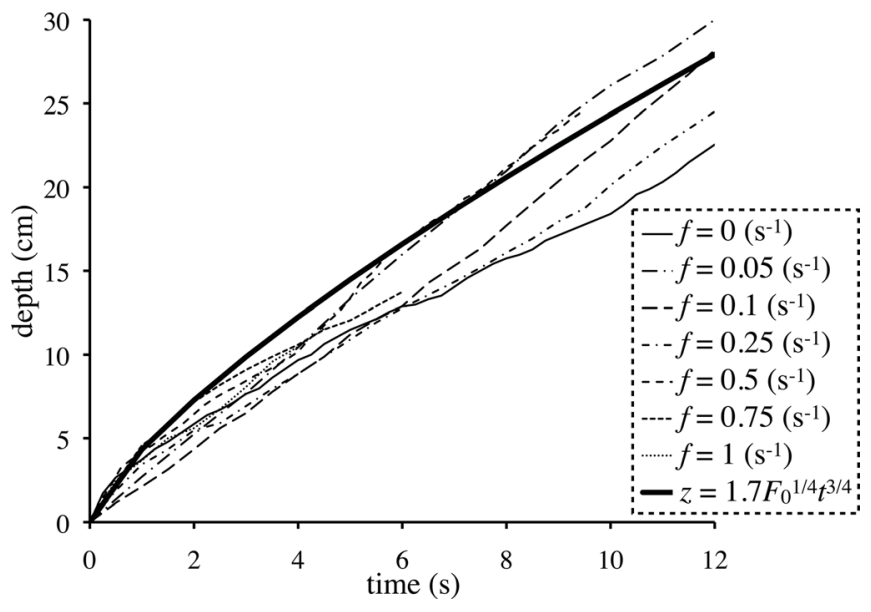

FIG. 4. Depth of the plume's front as a function of time for $t<t_{r}=3 T_{f} / 4$ Thick line denotes $z=1.7 F_{0}^{1 / 4} t^{3 / 4}\left(\right.$ Fernando et al. $\left.{ }^{6}\right)$ and the other lines are the experimental results for various rotation rates.

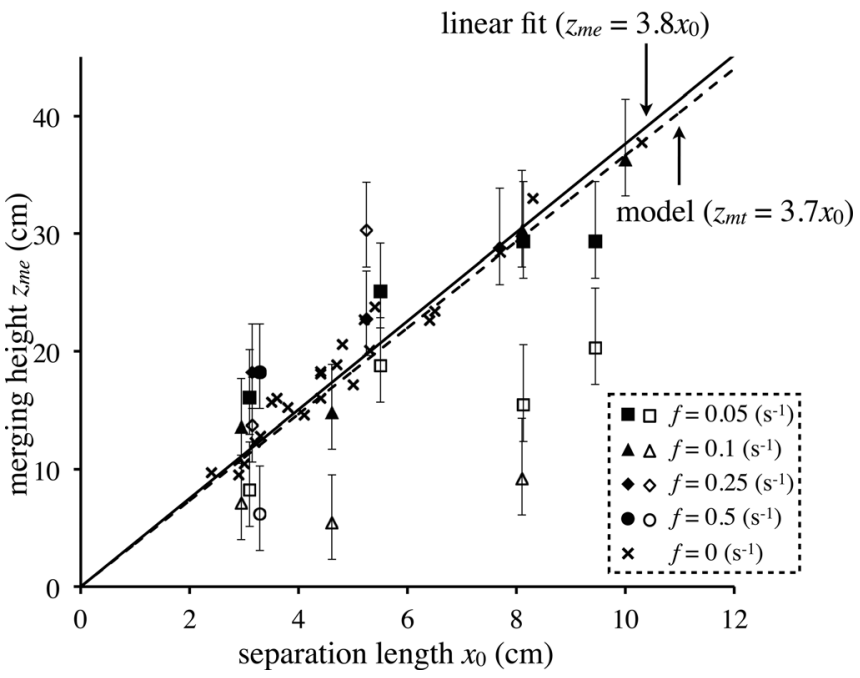

FIG. 5. Experimentally measured plume merging height $z_{m e}$ plotted against the separation length $x_{0}$ for rotating experiments at early times $T_{f} / 2$ $<t_{m}<3 T_{f} / 4$ (plotted with solid symbols) and for later times $3 T_{f} / 4<t<T_{f}$ (plotted with open symbols). The range of errors is $+4.1 /$ $-3.1 \mathrm{~cm}$ for $x_{0} \leq 6 \mathrm{~cm}$ and $+5.1 /-3.1 \mathrm{~cm}$ for $x_{0}>6 \mathrm{~cm}$, which is estimated from the sum of the standard deviation of $z_{m e}$ and a one-sided error of boxaveraging. As written in the text, the value of the standard deviation is based on non-rotating experiments, so these error bars are the minimum estimation for rotating experiments. The solid line is the best linear fit of the experimental data at early times. For comparison, the merging heights for nonrotating experiments are shown with cross signs, and the solid line shows the theoretical prediction (Eq. (4)) for non-rotating flows. Merging heights for $\left(f, x_{0}\right) \approx(0.25,10),(0.5,8),(0.5,10),(0.75,5),(0.75,8),(0.75,10)$ and $f=1 \mathrm{~s}^{-1}$ are not shown because the plumes are sufficiently "distant" for merger never to be observed in our experiments. The merging heights for $\left(f, x_{0}\right) \approx(0.5,5),(0.75,3)$ are also not shown as they were difficult to determine experimentally, while for the later time experiments the merging heights $z_{m e}$ for $\left(f, x_{0}\right) \approx(0.1,10),(0.25,8)$ are not shown because the plumes became separated due to the influence of rotation, and so no longer merged.

standard deviation for the non-rotating experiments. Here, the standard deviation of $z_{m e}$ is based on non-rotating experiments with time-averaging over $30 \mathrm{~s}\left(\approx T_{f} / 4\right.$ for $\left.f=0.05 \mathrm{~s}^{-1}\right)$, which nevertheless over such short times should behave in a very similar fashion to rotating experiments. Because of the relatively short time-averaging, unsurprisingly the standard deviation is substantially larger, approximately $3.1 \mathrm{~cm}$, than in the cases discussed above with 120 s averaging time. Indeed, it is reasonable to suppose that the standard deviation for rotating experiments over perhaps even shorter averaging periods could be larger than $3.1 \mathrm{~cm}$, so this value should be considered as a minimum estimation of the error bars.

Naturally, for all these experiments the initial merger height $z_{m e}<z_{r}$, and so these plumes are sufficiently "close" to behave like the non-rotating case. Indeed, since for these plumes by construction the leading-edge front has not yet reached the height at which rotation becomes significant, the merger continues to take place in the conical region of the plume behind the front. As a reference, we also plot with crosses the relationship between $z_{m e}$ and $x_{0}$ for the non-rotating experiments shown in Fig. 3. In this early time interval, it is clear that $z_{m e}$ has the same dependence on $x_{0}$ as in the non-rotating experiments, which is not surprising, since from Fig. 4, the plume dynamics are largely unaffected by rotation. As noted on the caption, however, sufficiently "distant" 
plumes in flows with high rotation rates had not merged by this time $t_{r}$. The solid line in Fig. 5 shows the best linear fit $\left(z_{m e}=3.8 x_{0}\right)$ to the data from rotating experiments (plotted with solid symbols) at sufficiently early times to be largely unaffected by rotation. Similarly to the result found for the non-rotating experiments, the difference between the best linear fit and the model $\left(z_{m t}=3.7 x_{0}\right)$ is consistent with an uncertainty of approximately $10 \%$ in the entrainment constant. An alternative, yet equivalent way to express the fact that the experimental evidence points to a relatively small variability in the implied value of the entrainment constant is to consider $\alpha$ as a fitting parameter in (Eq. (4)). Doing this, we obtain $\alpha=0.113$ for non-rotating experiments and $\alpha=0.116$ for rotating experiments at early times $t<t_{r}$. These values of $\alpha$ are within $10 \%$ of $\alpha=0.12$ which we determined by the completely independent "filling box" technique $^{17}$ for a single plume from the same source. Therefore, we believe that the entrainment constant is not affected considerably by either the nearby plume or the rotation at sufficiently early times.

We also plot on the same figure with open symbols the relationship between the "apparent" value of $z_{m e}$ (as determined by our method) and $x_{0}$ for later times $3 T_{f} / 4<t<T_{f}$ for the same plumes. In this later time interval, $z_{m e}$ is strongly affected by rotation, and is modified from its initial value, but there is no categorical relation between $z_{m e}$ and $x_{0}$, although in most cases the merger height is reduced. As we discuss in more detail below, this is due to the influence of a variety of competing physical effects due to the flow rotation, as well as the possibility that the merger was apparent rather than real due to the side-view optical method we have used to determine merger. Therefore, it is appropriate to treat any conclusions drawn from this late-time data with caution. Finally, it is important to appreciate that there were still a large number of experiments (with sufficiently "distant" source conditions) that did not undergo even apparent merger in this time interval.

An interesting quantity to consider is the root-meansquare error between the observations and the non-rotating theory given by (Eq. (4)), defined as

$$
e_{\mathrm{rms}}=\sqrt{\frac{\sum_{i}^{M}\left(z_{m e}^{i}-z_{m t}^{i}\right)^{2}}{M}},
$$

where $M$ is the number of experiments, and a superscript $i$ denotes the $i$ th experiment. For the non-rotating experiments, $e_{\text {rms }}=1.87 \mathrm{~cm}$, while for the early-time data from the rotating experiments with $T_{f} / 2<t_{m}<3 T_{f} / 4, e_{\mathrm{rms}}=2.89 \mathrm{~cm}$, and for the late-time rotating experiments with $3 T_{f} / 4<t<T_{f}$, $e_{\mathrm{rms}}=10.98 \mathrm{~cm}$.

From the above analysis, we deduce that the effect of rotation becomes important after $t \approx 3 T_{f} / 4$, in agreement with the result of Fernando et al. ${ }^{6}$ Of course, as time continues, the flow becomes strongly influenced by rotation, and even if the "close" plumes have merged "early," the rotation still strongly modifies the subsequently observed merger height. On the other hand, if the plumes have not merged by the critical time at which rotation becomes important, the non-rotating model is essentially irrelevant to any aspect of the flow's development. Furthermore, our experimental results show that the merging height for $t<3 T_{f} / 4$ agrees with (Eq. (4)), i.e. the theoretical model of Kaye and Lin$\operatorname{den}^{11}$ for non-rotating plumes. This implies that for $t<t_{r}=3 T_{f} / 4$ the entrainment constant $\alpha$ does not vary significantly from $\alpha$ in a non-rotating fluid. It is unsurprising that the rms error is slightly larger for the early-time rotating experiments than for the non-rotating experiments, because there is likely to be some (relatively small) effect of rotation, or indeed an effect of relatively shorter time-averaging of the analysed images for the rotating experiments.

\section{Rotating flow characteristics}

To understand the behavior of the plumes when they are affected by rotation, it proved useful to consider flow visualization. Two qualitatively different behaviors were observed for "close" (i.e., plumes which merged before the time $t_{r}$ at which rotation became important) and "distant" plumes. As an example of a rotating experiment for "close" plumes, we show the evolution of two plumes and the flow induced by the plumes at late time for $f=0.25 \mathrm{~s}^{-1}, x_{0}=5.2 \mathrm{~cm}$ in Figs. 6 and 7(a), respectively, each taken from within the rotating experimental frame. It is apparent that at early times [panels 6(a) and 6(b)] the plumes merge, and also that the merger continues to occur at the conical plume-like section, rather than exclusively at the thermal-like leading edge of the plume.
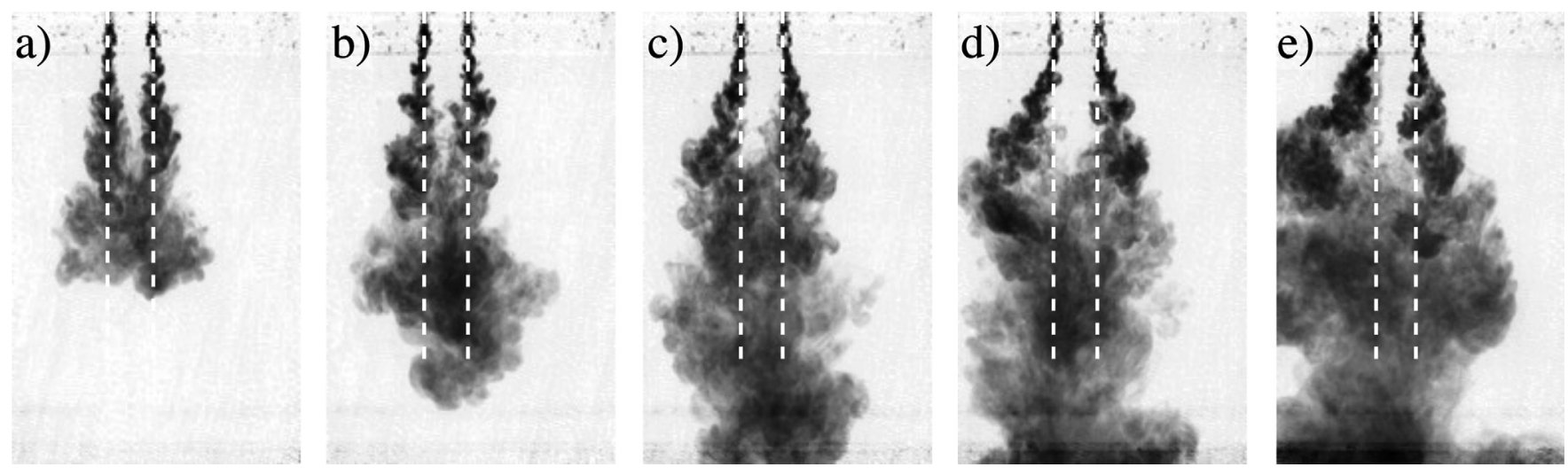

FIG. 6. Side-view dye concentration of two "close" plumes with $f=0.25 \mathrm{~s}^{-1}, x_{0}=5.2 \mathrm{~cm}$ at $t=$ (a) $0.4 T_{f}$, (b) $0.6 T_{f}$, (c) $0.8 T_{f}$, (d) $T_{f}$, and (e) $1.2 T_{f}$. Here $T_{f}=2 \pi / f$ is the inertial period. White dashed lines show the vertical. 

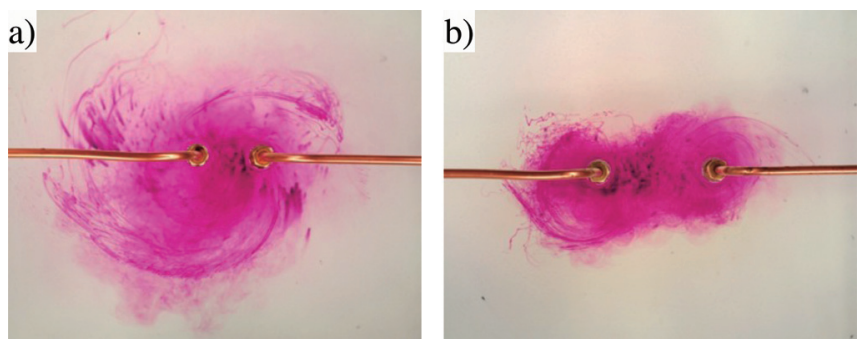

FIG. 7. (Color online) (a) Top-view photograph showing a vortex generated by "close" plumes with $f=0.25 \mathrm{~s}^{-1}, x_{0}=5.2 \mathrm{~cm}$ at $t=2.2 T_{f}$. (b) Topview photograph showing two distinct vortices generated by "distant" plumes with $f=0.75 \mathrm{~s}^{-1}, x_{0}=8.4 \mathrm{~cm}$ at $t=2.2 T_{f}$. In each image, we used purple potassium permanganate crystals to dye the water in the tank.

Interestingly, it is apparent that at later times (in panels 6(c) and 6(d)) for $t \geq 0.8 T_{f}$ (and certainly after initial merger) the axes of the plumes tilt away from the vertical and a single vortex is generated at the midpoint between the plumes' sources. This vortex is induced by the entrainment effect of the combined plumes, entraining fluid from greater radial distances from the rotation axis. The Coriolis force then deflects this flow to form a cyclonic vortex, centered over the rotation axis, unlike the plumes, each of which has a source some distance from the intersection of the rotation axis and the free surface. This single vortex is clearly apparent in Figure 7(a), taken at time $t=2.2 T_{f}$. Therefore, the later-time developing vortical flow inevitably tilts the plumes, in a fashion analogous to the well-known effect of cross-flows on rising plumes. ${ }^{18}$

Indeed, because of the relative locations of the plume sources and the vortex, the two plume sources are typically deflected in a spiral braid-like structure, as shown in Fig. 8. The rotation is weaker in this case than in the case shown in Figs. 6 and 7(a), and so the tilt away from vertical is not as pronounced. The plumes definitely merge before rotation significantly affects the dynamics, and a single cyclonic vortex develops. However, by careful analysis of the experimental videos, it is apparent that the two plumes "braid" under the effect of this developing cyclonic vortex. From this side view, the vortex flows from left to right in the foreground, and from right to left in the background. The plume initially on the left (whose axis' approximate location is marked with a solid white line) is advected by this vortex from left to right in front of the plume initially on the right (marked with a dashed line) and then in turn the initially left-located plume is advected from right to left behind the initially rightlocated plume. As discussed in more detail below, such braiding can make it extremely difficult to identify a true merger height at later times when "close" plumes are strongly affected by rotation. In particular, it is very important to appreciate that the values of $z_{m e}$ at later times shown by open symbols in Fig. 5 were determined by using the sideview-based method discussed above. Therefore, these values do not necessarily correspond to a "true" merging height, especially for braiding cases where a single peak in light intensity (used to identify an apparent merger height) may well be associated merely with the braiding plumes passing by each other along the line of sight of the camera.

Conversely, Fig. 7(b) shows the flow induced by the plumes for $f=0.75 \mathrm{~s}^{-1}, x_{0}=8.4 \mathrm{~cm}$ [at the same late time $t=2.2 T_{f}$ as for the close plumes shown in Fig. 7(a)] which exhibits the other qualitatively different behavior occurring when the plumes' source separation is relatively "distant." In this experiment, two vortices are generated (appearing after $t>t_{r}=3 T_{f} / 4$ ), due to the entrainment into each plume independently. Each plume still has a distinguishable associated flow, and so the Coriolis force generates independent cyclonic vortices. After $t \sim 6 T_{f}$ the vortices started shedding from the sources (not shown) and neither the plumes nor the vortices merged, but remained distinct. In all experiments, the plumes did not reach a steady state because the plumes were advected by the vortex or vortices generated by the plumes themselves. The number of vortices generated in each experiment is shown in Table II. A trend is clearly apparent: increasing either separation distance or rotation rate makes it more likely for two vortices to develop.

\section{E. Vortex generation}

We therefore propose the following physical interpretation (consistent with our experimental observations) for the processes which control the generation of either a single vortex or two vortices. When the rotation rate is low and the separation length is small, and so the plumes are "close," the
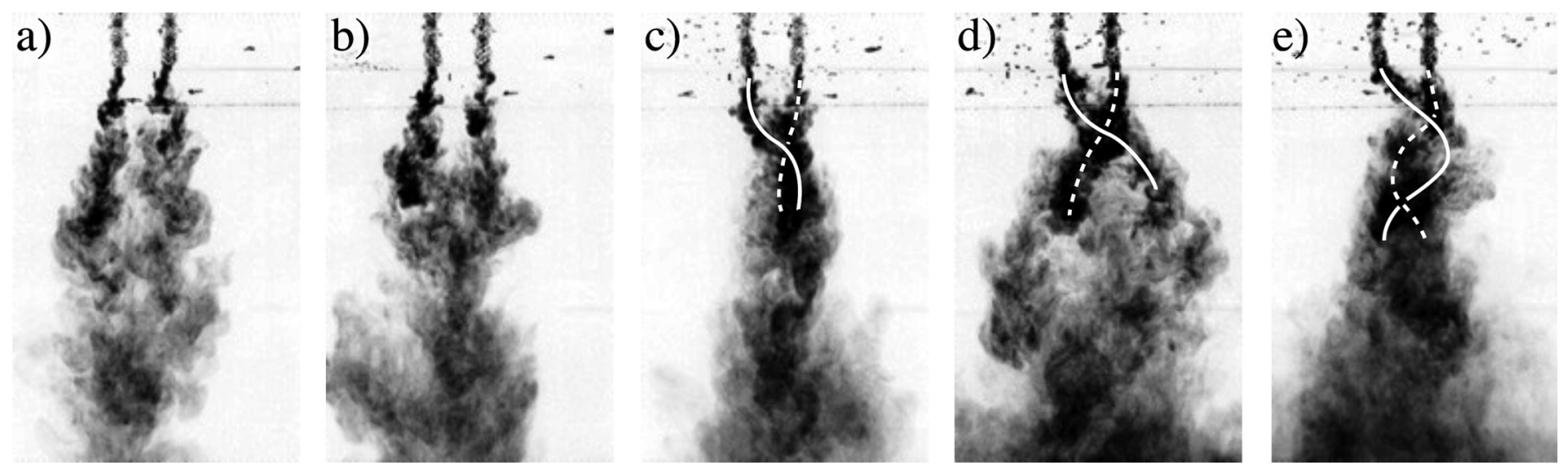

FIG. 8. Side view dye concentration of two "close" plumes with $f=0.1 \mathrm{~s}^{-1}, x_{0}=4.6 \mathrm{~cm}$ at $t=$ (a) $0.4 T_{f}$, (b) $0.6 T_{f}$, (c) $0.8 T_{f}$, (d) $T_{f}$, and (e) $1.2 T_{f}$ Here $T_{f}=2 \pi / f$ is the inertial period. The approximate locations of the plumes' axes are shown with solid (left source) and dashed (right source) lines, illustrating the way that rotation can lead to a "braiding" of the plumes at later times. 
TABLE II. Number of vortices generated by the plumes. Number 1 denotes that a single vortex is generated at the midpoint between two plumes' sources and $\mathbf{2}$ denotes that two vortices were generated, one from each plume, centred above the location of each plume source.

\begin{tabular}{llllll}
\hline \hline & & \multicolumn{4}{c}{$x_{0}(\mathrm{~cm})$} \\
\cline { 3 - 6 } & & 3 & 5 & 8 & 10 \\
\hline & 0.05 & $\mathbf{1}$ & $\mathbf{1}$ & $\mathbf{1}$ & $\mathbf{1}$ \\
& 0.1 & $\mathbf{1}$ & $\mathbf{1}$ & $\mathbf{1}$ & $\mathbf{1}$ \\
& 0.25 & $\mathbf{1}$ & $\mathbf{1}$ & $\mathbf{1}$ & $\mathbf{2}$ \\
& 0.5 & $\mathbf{1}$ & $\mathbf{1}$ & $\mathbf{2}$ & $\mathbf{2}$ \\
& 0.75 & $\mathbf{1}$ & $\mathbf{2}$ & $\mathbf{2}$ & $\mathbf{2}$ \\
& 1 & $\mathbf{2}$ & $\mathbf{2}$ & $\mathbf{2}$ & $\mathbf{2}$ \\
\hline \hline
\end{tabular}

plumes are affected by rotation only after their initial merger (i.e., the plumes have merged before $t=t_{r}=3 T_{f} / 4$ when the rotational effects become important), and so a single vortex will be generated by the resulting single plume, as shown schematically in Fig. 9(a). It is very important to understand, however, that rotation has profound and varied effects on the plumes even in this case at sufficiently late times. These effects may lead to the non-systematic variability in the observed later-time merger heights (as shown on Fig. 5 with open symbols), because aspects of the flow's rotation both increase and decrease the tendency of the two plumes to merge.

As discussed in detail by Fernando et al., ${ }^{6}$ rotation reduces the descent speed since radial entrainment is suppressed in favour of vertical entrainment from fluid further (vertically) away from the source, but at the same radial distance from the rotation axis, thus avoiding large (and dynamically difficult) changes in angular momentum of fluid elements. This entrainment-related "suction" tends to cause the plume to slow relative to its behaviour in a non-rotating environment, and hence bulge outward, thus leading to a reduced height of merger, compared to the initial non-rotating merger height. We sometimes observed this "bulging" of the constituent plumes, thus reducing the height of merger, and indeed this seemed to be the most significant effect induced by rotation. However, it is
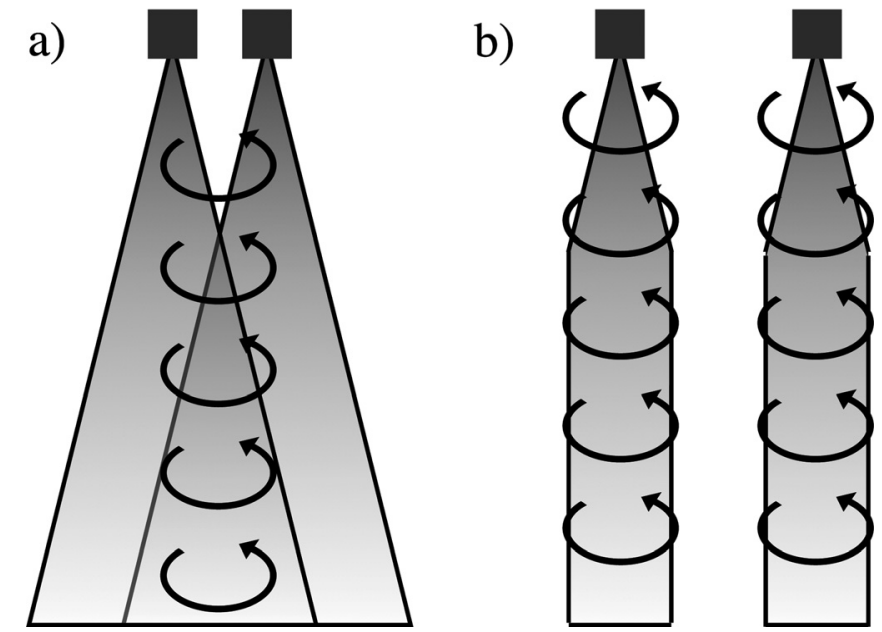

FIG. 9. Schematic illustration of the two plumes generating (a) a single vortex after merging when the sources are "close" and (b) two vortices before they have the opportunity to merge when the sources are "distant." very important to appreciate that there is every possibility that the images can be misleading, as they are two-dimensional side-views of a dynamically evolving flow. In particular, as noted above, and shown in Fig. 8, the developing vortex could induce a spiral "braiding" of the two plumes. Viewed from the side [perhaps most clearly illustrated by comparison of Figs. 8(d) and 8(b)], merger could appear to have occurred at a much smaller distance from the sources than at early times, when in fact the two plume conical sections remained largely distinct.

Indeed rotation could also suppress, or at least delay merger, for at least two, quite different reasons. First, particularly for later times (as also noted in Fernando et al. ${ }^{6}$ ), rotation suppressed entrainment throughout the plume, thus sometimes leading to thinner plumes at a given height, and so a tendency for merger only to occur at a greater height. Secondly, and perhaps more significantly, as noted above, the spiral tilting and braiding of the two plume sources could actually lead to an enhanced separation of the two plumes, thus leading to an increase in the merger height from its initial value. This latter effect could even be accentuated in certain circumstances when the vortex migrated away from its formation location directly over the rotation axis, as is slightly apparent on Fig. 7(a). The somewhat greater tilt shown for the left plume in Fig. 6 is due principally to the fact that the developing vortex migrated slightly to the left away from being centred precisely over the rotation axis of the system (not shown).

On the other hand, when the rotation rate is high and the separation length is large (and so the plumes are "distant" in our terminology), the plumes feel the effects of rotation before they have the opportunity to merge and two vortices are generated, one for each plume as shown schematically in Fig. 9(b), and so over the time scale of our experiments merger of the plumes is completely suppressed. The independent entrainment processes and subsequent deflection by the Coriolis force for each plume thus lead to the development of a vortex, and the two plumes evolve separately, even as the influence of rotation becomes important. [We note that in our experiments a single vortex never resulted from the merging of two vortices. This is because the time scale of the merging of vortices $\left(t \sim 20 T_{f}\right)$ (Ref. 19) is much longer than the time scale on which we focus here $\left(t \sim T_{f}\right)$.]

In light of the above discussion, we now have a way to predict the number of vortices generated by the two plumes. If the plumes are "close" and so their initial predicted merger height $z_{m t}$ (as defined in (Eq. (4)); Ref. 11) is less than the height $z_{r}$ (as defined in (Eq. (3)); Ref. 6) at which rotation becomes important, we expect a single vortex to be formed. On the other hand, if $z_{m t}>z_{r}$, and so the plumes are sufficiently "distant" for merger not to have occurred before rotation becomes dynamically important, we expect to observe two vortices. This prediction is consistent with the data. In Fig. 10, we plot various experiments in the $z_{m t}-z_{r}$ plane, using different symbols to denote the number of observed vortices. The line denoting $z_{r}=z_{m t}$ effectively separates the two qualitatively different types of behavior. Therefore, we can predict the number of vortices generated by the two plumes in terms of a critical separation distance $x_{c}$ from the definitions for $z_{r}$ (Eq. (3)) and $z_{m t}$ (Eq. (4)) using the 


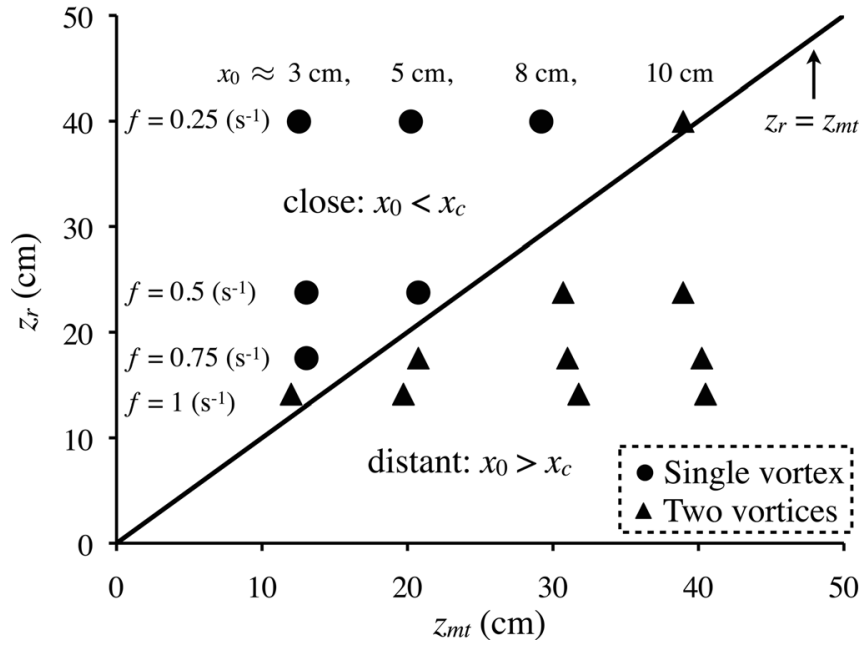

FIG. 10. Number of vortices generated by the plumes shown in the $z_{m t}-z_{r}$ plane. Circles denote single vortex and triangles denote two vortices. Solid line denotes $z_{r}=z_{m t}$. The results for $f=0.05,0.1 \mathrm{~s}^{-1}$ are not shown because $z_{r} \gg z_{m t}$ for these experiments and a single vortex was generated for every $x_{0}$.

(specific) buoyancy flux $F_{0}$, Coriolis parameter $f$, and the entrainment constant $\alpha$. Two vortices are expected to occur if $z_{m t}>z_{r}$, or equivalently if $x_{0}>x_{c}$ where the critical separation distance $x_{c}$ is defined as

$$
x_{c}=\frac{25 \alpha}{2} F_{0}^{1 / 4} f^{-3 / 4} .
$$

In summary, this is a way in which the inherent time-dependence of the evolving flow manifests itself, and enters into the physical evolution. There is an early time dynamic for "close" plumes, which means that the plumes actually form a merged, combined structure that is then influenced by rotation. If the plumes are sufficiently "distant" for the merger not to occur before the onset of the influence of rotation, then the individual vortices (each associated with the separately evolving plume) actually themselves strongly suppress any future likelihood of merger.

\section{CONCLUSIONS}

We carried out laboratory experiments on two coalescing axisymmetric turbulent plumes in both non-rotating and rotating, homogeneous fluids. For the non-rotating experiments, our results, as shown in Fig. 3, agree well with the theory of Kaye and Linden ${ }^{11}$ predicting a theoretical merging height $z_{m t} \approx(0.44 / \alpha) x_{0}$, where $x_{0}$ is the initial separation length of the two plumes' sources. For the rotating experiments, our results show that for $t<t_{r}=3 T_{f} / 4$ the experimentally observed merging height $z_{m e} \approx z_{m t}$ for sufficiently "close" plumes, where $x_{0}<x_{c}$ as defined in (Eq. (9)), and so they agree with the theory of Kaye and Linden, ${ }^{11}$ which does not account for rotation. However, for $t>t_{r}$, the plumes were strongly affected by various aspects of the flow associated with the system rotation, in particular by the altered entrainment dynamics and the single vortex which developed, and so typically $z_{m e}$ did not continue to agree with $z_{m t}$ for $t>t_{r}$. That the effect of rotation became significant for $t>t_{r}=3 T_{f} / 4$ is in agreement with the work of Fernando et $a l .{ }^{6}$ who considered the development of a single axisymmetric turbulent plume in a rotating, homogeneous fluid, and we also observed that the evolution of the leading front of the plume was consistent with their observations.

Indeed, both the early- and late-time evolution of the interacting plumes can be interpreted as consequences for neighbouring evolving plumes of various aspects of the rotational effects on plumes discussed in their paper. There is in essence a "race": can the plumes evolve toward merger (which happens later and later the further apart they are), before rotation can strongly influence the flow dynamics? Therefore, plumes have to be sufficiently close together to merge, since if they are not, their notional (non-rotating) time to initial merger is too long, so the effects of rotation can "catch up" and hence suppress merger. Furthermore, the subsequent, later-time dynamics of the rotating experiments is strongly affected by the vortices induced by the plumes. For "close" plumes, a single vortex develops from the merged plumes, while for more "distant" plumes we observe two vortices. The criterion to determine the occurrence of two vortices is physically that merger does not take place before rotation becomes important: this is equivalent to $z_{m t}>z_{r}$, or equivalently $x_{0}>x_{c}$ as defined in (Eq. (9)).

As a final observation, it is important to appreciate that there is a non-trivial difference between the flow regime which we consider, and that considered previously by Helfrich and Battisti. ${ }^{15}$ Though both their study and the present experiments showed that two sufficiently nearby plumes in a rotating fluid generate a single vortex, the dynamics controlling the generation of a single vortex in Helfrich and Battisti $^{15}$ are different from that in the present experiments, as our plume sources were typically much closer together than in their study. In Helfrich and Battisti, ${ }^{15}$ a single vortex was generated by the merging of the two vortices developed by each plume. This process can be seen in Fig. 7 of Helfrich and Battisti, ${ }^{15}$ and the time scale was $t \sim 14 T_{f}$, which is comparable to the time scale $\left(t \sim 20 T_{f}\right)$ of merging of vortices in Griffiths and Hophinger. ${ }^{19}$ On the other hand, the time scale $\left(t \sim T_{f}\right)$ which we focused on is appreciably quicker than the time scale necessary for the merging of the vortices, and the vortices were not observed to merge in our experiments. In the present experiments, a single vortex was generated after the merging of two (very) "close" plumes as explained above, hence highlighting a novel mechanism for a single vortex generation from two "close" plumes (in the well-defined sense that their source separation $x_{0}<x_{c}$ $\left.=(25 \alpha / 2) F_{0}^{1 / 4} f^{-3 / 4}\right)$ in a rotating fluid.

\section{ACKNOWLEDGMENTS}

We thank all staff and fellows of the Geophysical Fluid Dynamics Program at the Woods Hole Oceanographic Institution in 2009 and, in particular, Antonello Provenzale for initial discussions inspiring the laboratory experiments. The comments of three anonymous referees have substantially improved the clarity of this paper and their constructive criticism is gratefully acknowledged. This work was supported by the Center for Planetary Science. 
${ }^{1}$ A. W. Woods, "Turbulent plumes in nature," Annu. Rev. Fluid Mech. 42, $391(2010)$

${ }^{2}$ B. R. Morton, G. R. Taylor, and J. S. Turner, "Turbulent gravitational convection from maintained and instantaneous sources," Proc. R. Soc. London, Ser. A 234, 1 (1956).

${ }^{3}$ J. S. Turner, Buoyancy Effects in Fluids (Cambridge University Press, Cambridge, 1973).

${ }^{4}$ C. P. Caulfield and A. W. Woods, "Turbulent gravitational convection from a point source in a non-uniformly stratified environment," J. Fluid Mech. 360, 229 (1998).

${ }^{5} \mathrm{P}$. F. Linden, "Convection in the environment," in Perspectives in Fluid Dynamics, edited by G. K. Batchelor, H. K. Moffat, and M. G. Worster (Cambridge University Press, Cambridge, 2002).

${ }^{6}$ H. J. S. Fernando, R.-R. Chen, and B. A. Ayotte, "Development of a point plume in the presence of background rotation," Phys. Fluids 10, 2369 (1998).

${ }^{7}$ J. S. Turner, "The 'starting plume' in neutral surroundings," J. Fluid Mech. 13, 356 (1962).

${ }^{8}$ M. M. Scase, A. J. Aspden, and C. P. Caulfield, "The effect of sudden source buoyancy flux increases on turbulent plumes," J. Fluid Mech. 635, 137 (2009).

${ }^{9}$ J. W. M. Bush and A. W. Woods, "Vortex generation by line plumes in a rotating stratified fluid,” J. Fluid Mech. 388, 289 (1999).
${ }^{10}$ C. Y. Ching, H. J. S. Fernando, L. A. Mofor, and P. A. Davies, "Interaction between multiple line plumes: A model study with applications to leads," J. Phys. Oceanogr. 26, 525 (1996).

${ }^{11}$ N. B. Kaye and P. F. Linden, "Coalescing axisymmetric turbulent plumes," J. Fluid Mech. 502, 41 (2004).

${ }^{12}$ C.-C. P. Caulfield and A. W. Woods, "Plumes with nonmonotonic mixing behaviour," Geophys. Astrophys. Fluid Dyn. 79, 173 (1995).

${ }^{13}$ G. R. Hunt and N. G. Kaye, "Virtual origin correction for lazy turbulent plumes," J. Fluid Mech. 435, 377 (2001).

${ }^{14}$ M. K. Tivey, "Generation of seafloor hydrothermal vent fluids and associated mineral deposits," Oceanography 20, 50 (2007).

${ }^{15}$ K. R. Helfrich and T. M. Battisti, "Experiments on baroclinic shedding from hydrothermal plumes," J. Geophys. Res. 96, 12511, doi: 10.1029/ 90JC02643 (1991).

${ }^{16} \mathrm{H}$. Yamamoto, "Laboratory experiments on two coalescing axisymmetric turbulent plumes in a rotating fluid," Woods Hole Oceanog. Inst. Tech. Rept. WHOI-2010-01 (Woods Hole Oceanog. Inst., Woods Hole, Mass., 2010).

${ }^{17}$ W. D. Baines, "A technique for the direct measurement of volume flux of a plume," J. Fluid Mech. 132, 247 (1983).

${ }^{18} \mathrm{P}$. R. Slawson and G. T. Csanady, "On the mean path of buoyant, bentover chimney plumes," J. Fluid Mech. 28, 311 (1967).

${ }^{19}$ R. W. Griffiths and E. J. Hopfinger, "Coalescing of geostrophic vortices," J. Fluid Mech. 178, 73 (1987). 